\title{
Fast and Accurate Thickness Mapping of Thin Liquid Films
}

\author{
Zhe Wang ${ }^{1,2 *}$, Vincenzo Ferraro ${ }^{3}$, Biagio Mandracchia ${ }^{1}$, Ernesto Di Maio ${ }^{3}$, Pier Luca Maffettone ${ }^{3}$ and Pietro Ferraro ${ }^{1}$ \\ ${ }^{1}$ CNR-ISASI, Istituto di Scienze Applicate e Sistemi Intelligenti «E. Caianiello» del CNR, Via Campi Flegrei 34, 80078 Pozzuoli, \\ Napoli, Italy. \\ ${ }^{2}$ College of Applied Sciences, Beijing University of Technology, 100124 Beijing, China. \\ ${ }^{3}$ Dipartimento di Ingegneria Chimica, dei Materiali e della Produzione Industriale, Università di Napoli Federico II, Piazzale Tecchio 80 , \\ 80125 Napoli, Italy.
}

\begin{abstract}
The thickness of thin liquid films is of great interest to industrial processes and life science. However, there are not appropriate quantitative experimental tools for an adequate study of film evolution in case of not-ideal conditions. Here, we show the application of a holographic system for the evaluation of the 3D topography and thickness of evolving protein films. We use a custom holographic microscope that combines quantitative phase imaging with materials engineering. This technique offers an unprecedented level of details and we anticipate that it will promote a deeper understanding of the underlying physics of thin film dynamics.
\end{abstract}

\section{Introduction}

Thin liquid films flows are of fundamental importance in numerous areas of engineering, geophysics and biophysics $[1,2]$. These films display unusual dynamic behaviours in drainage and measurement of the thin fluid film thickness evolution during drainage is key to understand such phenomena [3, 4]. In turn, such knowledge can be utilized to design new materials, to guide technologies involving bubbles and to control biological functions involving bubbles and bubbles rupture. Nevertheless, there are not appropriate quantitative experimental tools for an adequate study of film evolution in case of not-ideal conditions.

Herein, we tackle this problem using a holographic technique that combines quantitative phase imaging with a custom setup designed to form and manipulate bubbles [5]. digital holography (DH) is often used in sensors and microscopes to assess small variations of concentration [6], cell mass profiling [7] or thickness in transparent samples below the diffraction limit [8, 9]. In particular, we use transmission digital holography (TDH) to directly observe protein films ranging from a thickness of $\mathrm{H} \approx 25 \mu \mathrm{m}$ down to the wavelength of a coherent laser source $(\mathrm{H} \approx 632.8 \mathrm{~nm})$. We report our findings on the local film thickness and surface structure of films determined by TDH and discuss the implications of using this optical technique for studying non-uniform transparent surfaces.

\section{Materials and Methods}

\subsection{Materials}

The protein films were based on an aqueous solution of egg white albumen (EWA). EWA came from whole eggs purchased at the local market. The percentage of solid content of EWA was measured before use. Only EWA with $14 \%$ solid content was used for our experiments. The solvent used to dilute our protein mixture was DPBS, provided by Sigma.

\subsection{Experimental setup}

The setup consists of an off-axis Mach-Zhender interferometer and engineered for in order to allow the formation of thin liquid films and to control the bubble growth, see Figure 1. The films were formed on top of a metal pipe with a side inlet that was connected to a syringe pump. The illumination source was a HeNe laser ( $\lambda=$ $632.8 \mathrm{~nm}$ ). Indeed, using a laser source to illuminate a thin-film could have some effects like dehydration. However, in most case, the laser power used for thin-film holographic recording is very weak $(<0.5 \mathrm{mw})$, therefor the dehydration caused by laser recording is negligible compared with the dehydration caused by the overall temperature and humidity of the environment.

In the Mach-Zehnder interferometer, the laser beam is divided in two parts by the first beam-splitter cube $\left(\mathrm{BS}_{\mathrm{t}}\right)$. The resulting beams are referred as object and reference beam. The object beam illuminates the sample from the top and forms the image on the camera. On the contrary, the reference beam goes towards the camera without encountering the sample. The two beams are collected by a second beam-splitter cube $\left(\mathrm{BS}_{2}\right)$. The cube is slightly tilted so that the two beams overlap with a small angle. This controls the period of the interference fringes, which can be adjusted according to the sampling requirements. Since the protein films usually have a complex surface structure, the Fresnel Hologram recording system was

\footnotetext{
* Corresponding author: z.wang@isasi.cnr.it
} 
applied. Lens $L_{1}$ and lens $L_{2}$ form a $4 F$ system to collect light beams.

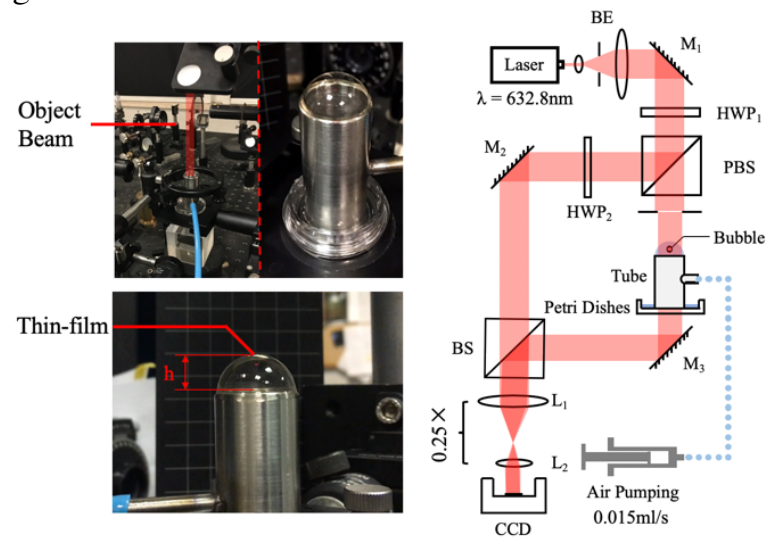

Fig. 2. Schematic of the transmission digital holography (TDH) setup that was used for this work. A laser source emits coherent single wavelength light into a beam expander (BE) and is then redirected by a mirror $\left(\mathrm{M}_{1}\right)$. The light passes through a halfway plate (HWP1) and is split into two beams by first beam-splitter cube (BS). The reference and object beams are collected by second bean-splitter cube (BS) and pass through two lenses ( $\mathrm{L}_{1}$ and $\mathrm{L}_{2}$ ) before being collected by a CCD or CMOS for holographic numerical reconstruction processing.

\section{Experimental results}

We have studied the growth and drainage of protein liquid bubbles formed from diverse dilutions of EWA. We examined six different dilutions $(5 \%, 20 \%, 40 \%, 60 \%$, $80 \%$, and $100 \%$ ) and for each dilution we performed 5 experiments. Each bubble was grown following the same protocol. First, the bubble was created on top of the metal pipe, see Figure 1. Once a stable film was formed the laser shutter was open and the recording begun. At the same time, the syringe pump started inflating air through the inlet and the bubble was let grow for 30s. At this point the bubble was let drain and observed for around 3 minutes, see Figure 2.

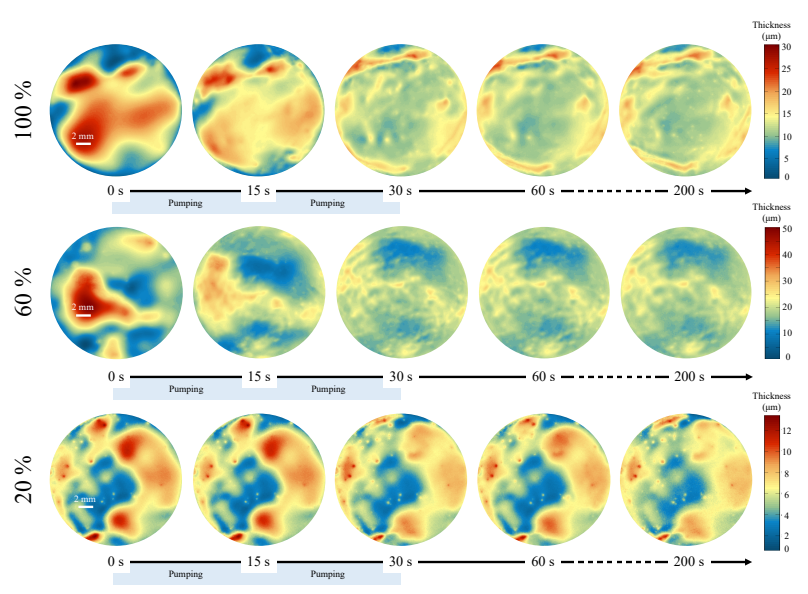

Fig. 2. Bubble thickness variation with time. Phosphate buffered saline (PBS) was mixed with egg white to make the experimental samples, and, pumping air at $0.015 \mathrm{ml} / \mathrm{s}$ to help bubble film grow. Each set of experiments lasted $200 \mathrm{~s}$, pumping air for the first 30 seconds, then stopped. The figure shows, from top to bottom, the thickness distribution of $100 \%$ egg white film, $60 \%$ egg white film and $20 \%$ egg white film.

In general, EWA bubbles showed a high level of stability. The drainage was mainly limited to the first seconds of the pumping phase, after which the system reaches an equilibrium. However, this equilibrium does not correspond to a homogeneous distribution of the proteins along the bubble's surface. Indeed, after 200s it is still possible to observe macro areas of protein aggregates all along the surface of the bubbles at every concentration.

\section{Conclusions}

In conclusion, we proposed a system that gathers all the features required to study evolving liquid thin film. First, the spatial resolution is limited only by the optics used. Second, it gives the full-field three-dimensional information of the sample, unlike techniques that use photomultipliers to have very fast measurement but only in one point. Third, $\mathrm{DH}$ does not require multiple exposures and can be matched with high-speed cameras to measure rapidly changing features, such as the rim of the hole formed by the rupture of the liquid film.

Finally, our study suggests that the use of EWA protein solutions to cast bubble films results in unique features that were not previously observed when detergents are used. Moreover, liquid films made from detergents are typically uniform and at a thickness range suitable for analysis by interferometry. On the other hand, our protein films do not show interference patterns owing to a complicated surface structure causing light scattering and are tens of microns thick, which would not result in interference patterns for visible wavelengths.

\section{References}

[1] P. Marmottant and S. Hilgenfeldt, Nature 423, 153156 (2003);

[2] A. Salerno, E. Di Maio, S. Iannace, and P. A. Netti, J. Porous Mater. 19, 181-188 (2012);

[3] L. Champougny, M. Roché, W. Drenckhan, and E. Rio, Soft Matter 12, 5276-5284 (2016) ;

[4] D. Tammaro, R. Pasquino, M. M. Villone, G. D’Avino, V. Ferraro, E. Di Maio, A. Langella, N. Grizzuti, and P. L. Maffettone, Langmuir, 34, 5646-5654 (2018);

[5] B. Mandracchia, Z. Wang, V. Ferraro, M. M. Villone, E. Di Maio, P. L. Maffettone, and P. Ferraro, Light Sci. Appl 8, 20 (2019) ;

[6] F. Yesilkoy, R. A. Terborg, J. Pello, A. A. Belushkin, Y. Jahani, V. Pruneri, and H. Altug, Light Sci. Appl. 8, 17152 (2018);

[7] T. a Zangle and M. a Teitell, Nat. Methods 11, 12211228 (2014);

[8] B. Mandracchia, O. Gennari, V. Marchesano, M. Paturzo, and P. Ferraro, J. Biophotonics 10, 1163-1170 (2017);

[9] B. Mandracchia, O. Gennari, A. Bramanti, S. Grilli, and P. Ferraro, J. Biophotonics 11, e201700332 (2018). 